\title{
Treatment of respiratory syncytial virus infection with recombinant interferon alfa-2a
}

\author{
R Y T Sung, J Yin, S J Oppenheimer, J S Tam, J Lau
}

\begin{abstract}
A prospective randomised, double blind, controlled trial was conducted in 52 infants to determine whether recombinant interferon alfa-2a (INF- $\alpha-2 a)$ would reduce the morbidity of acute bronchiolitis and the respiratory syncytial virus shedding time. All infants had a positive direct antigen immunofluorescence test for respiratory syncytial virus. INF- $\alpha-2 a$ (50 $000 \mathrm{IU/kg/day)} \mathrm{or} \mathrm{placebo} \mathrm{was} \mathrm{admin-}$ istered by daily intramuscular injection for three consecutive days. Sixteen infants received INF- $\alpha-2 a$ and 36 received placebo treatment. The two groups were similar in demographic characteristics and initial oxygenation. The treatment group, however, had a significantly higher overall score for severity of illness at the start of treatment. More rapid drop of the clinical score was observed in the INF- $\alpha-2 a$ group after treatment in the first three days and the two groups had similar clinical severity by day 3 . There was no significant difference of the duration of viral shedding in the two groups. In conclusion, the overall clinical improvement was greater in the treatment group over the first three days, but the duration of viral shedding was not altered.

(Arch Dis Child 1993; 69: 440-442)
\end{abstract}

Respiratory syncytial virus is the most important cause of acute bronchiolitis and viral pneumonia in infants. In our experience in Hong Kong, outbreaks occur yearly with a summer peak between April and September and cause high morbidity. ${ }^{1}$ The sudden increase in admission rate often overstretches manpower and resources in the local hospitals and the potential for nosocomial spread always threatens other hospitalised infants with severe underlying problems such as congenital heart disease. $^{2}$

Efforts by previous investigators to develop a vaccine have so far been unsuccessful. ${ }^{3}$ Since the late $1980 \mathrm{~s}$ a broad spectrum antiviral agent, tribavirin (ribavirin), has been increasingly used for the treatment of severe respiratory syncytial virus infection in infants, mainly based on its clinical benefit in severe cases and minimal toxicity. ${ }^{4}$ However, its high cost and the labour intensive method of administration have prevented it from being used routinely for mild to moderately sick infants who form the bulk of patients. It is therefore unlikely that tribavirin will play an important part in shortening or aborting outbreaks.
In 1989, Isaacs and colleagues reported that both in vivo and in vitro production of interferon alfa (INF- $\alpha$ ) was impaired during acute respiratory syncytial virus bronchiolitis in infants and suggested the need for a therapeutic trial of INF- $\alpha$ in acute bronchiolitis. ${ }^{5}$ In the same year Taylor and colleagues measured INF- $\alpha$, infectious virus, and viral fusion protein in the nasopharyngeal secretions of 54 infants with acute bronchiolitis and reported that there was no significant correlation between INF- $\alpha$, virus, or viral fusion protein secretion and severity of illness or age of infant. However, it was shown in their results that only 26 of the 54 infants secreted significant amounts of INF- $\alpha .{ }^{6} \mathrm{~A}$ therapeutic trial of INF- $\alpha$ is therefore considered worthwhile because of its potential effect of inducing the resistance to virus replication in host cells. So far to our knowledge there has been only one study of INF- $\alpha$ in treatment of infants with respiratory syncytial virus bronchiolitis published in the English literature. ${ }^{7}$ This pilot study did not include a control group and it was therefore difficult to evaluate the efficacy of INF- $\alpha$. Nevertheless, it did show that as little as $10000 \mathrm{IU} / \mathrm{kg} /$ day induced a significant in vitro response in patients' mononuclear cells and that no toxicity was found. In view of the lack of information of the efficacy of $\mathrm{NF}-\alpha$, its relatively low cost compared with aerosolised tribavirin, and its easy administration, we decided to carry out a double blind, controlled study.

Patients and methods

Infants admitted to the paediatric wards at the Prince of Wales Hospital between April 1991 and October 1992 for the first time with acute bronchiolitis and a positive immunofluorescence test of the nasopharyngeal aspirate for respiratory syncytial virus were recruited. The diagnostic criteria for acute bronchiolitis adopted by the authors were: (i) age 24 months or younger, (ii) signs of preceding or coexisting viral respiratory illness, (iii) first attack of expiratory wheezing, and (iv) respiratory distress: dyspnoea or tachypnoea (respiratory rate $>40 / \mathrm{min}) .{ }^{8}$ Only those deemed ill enough to require at least three days of hospitalisation were enrolled. Patients with congenital heart disease or bronchopulmonary dysplasia were excluded.

The study was approved by the ethical committee of the Faculty of Medicine, the Chinese University of Hong Kong and consent was obtained from the parents of all patients. The patients were randomised and 
Demographic characteristics of enrolled infants and severity of illness at study entry. Figures are mean (SD) unless otherwise stated

\begin{tabular}{lccl}
\hline & $\begin{array}{c}\text { Placebo } \\
(n=36)\end{array}$ & $\begin{array}{c}\text { Treatment } \\
(n=16)\end{array}$ & $p$ Value $^{\star}$ \\
\hline Age (months) & $6.29(3.75)$ & $6.56(3.50)$ & NS \\
Weight (kg) & $7.28(1.64)$ & $7.98(1.79)$ & NS \\
Male/female & $26 / 10$ & $10 / 6$ & NS \\
$\begin{array}{l}\text { Days of respiratory syncytial virus symptoms } \\
\text { before treatment }\end{array}$ & $3.83(1.87)$ & $2.88(1.36)$ & NS \\
No of children requiring oxygen treatment & 14 & 8 & NS \\
SaO while breathing room air & $93.61(2.35)$ & $94.13(3.0)$ & NS \\
Fractional inspired oxygen (Vmin) & $0.42(0.62)$ & $0.59(0.83)$ & NS \\
Clinical score & $10.61(2.09)$ & $12.06(1.92)$ & 0.02 \\
\hline
\end{tabular}

*Wilcoxon rank sum test and Fisher's exact test; NS=not significant.

assigned to the treatment or control group in a ratio of $1: 2$.

Interferon alfa-2a (INF- $\alpha-2 a$; Roferon-A, Roche) was administered by daily intramuscular injection at a dosage of $50000 \mathrm{IU} / \mathrm{kg} /$ day for three days. The placebo consisted of the vehicle for INF- $\alpha-2 a$ and was given at the same volume and on the same schedule as the INF- $\alpha-2 a$ preparation. All patients received salbutamol at $0.15 \mathrm{mg} / \mathrm{kg} /$ dose and ipratropium at $125 \mu \mathrm{g} /$ dose in $2 \mathrm{ml}$ of saline by inhalation. Oxygen supplement was given when the oxygen saturation $\left(\mathrm{SaO}_{2}\right)$ measured by pulse oximeter was less than $92 \%$.

Initial and daily physical examinations were conducted by one investigator (RYTS) blind to the interferon treatment. The vital signs were checked every four hours by the nurses and the highest readings during the day and the night were put on to a computerised form. Ten daily signs and symptoms, namely nasal congestion, cough, chest retraction, hyperinflation, wheezing, rales, rhonchi, lethargy, irritability and anorexia, were rated on computerised forms from 0 (normal) to 3 (severe). The unweighted arithmetic sum of the assessed points yielded the patients' clinical severity score. A score of 0 represented normal value and the maximum possible score was 30 , which indicated most severe illness. Twice daily evaluation of $\mathrm{SaO}_{2}$ was conducted non-invasively with a pulse oximeter. The duration of the need for oxygen supplementation and daily fraction of inspired oxygen were recorded.

Specimens of nasopharyngeal aspirates were collected with a mucus extractor (Uno, Denmark) and flushed into a standard volume of virus transport medium once daily during the hospitalisation. Direct immunofluorescent antigen tests of respiratory syncytial virus and viral cultures for respiratory syncytial virus, influenza, parainfluenza, adenovirus, and enteroviruses on LLCMK2, MDCK, Hep-2, and human fetal lung cell lines ${ }^{1}$ were carried out daily. An aliquot of secretions in transport medium was saved and stored at $-70^{\circ} \mathrm{C}$ for enzyme linked immunosorbent assay (ELISA) of the presence of respiratory syncytial virus antigen (Ortho Diagnostic, NY). 9 The duration of virus shedding was defined as the period between onset of illness and the three detection methods showing negative results.

Samples of blood were obtained on admission and before discharge to monitor the complete blood count and hepatic transaminases.
Comparability between the INF- $\alpha-2 a$ and placebo treated groups was assessed using the Wilcoxon rank sum test and Fisher's exact test. The outcome measures including the duration of oxygen treatment, change in severity score between admission and day 1 , day 2, day 3 and changes in temperature, heart rate, and respiratory rate were also compared by the Wilcoxon rank sum test. The duration of virus shedding was compared by Kaplan-Meier's life table method and log rank test. The complete blood count and hepatic transaminases results were compared by Student's $t$ test.

\section{Results}

Fifty two infants with proved respiratory syncytial virus bronchiolitis were studied: 36 infants were randomised to receive placebo and 16 received $\mathrm{INF}-\alpha-2 \mathrm{a}$. The characteristics of the 52 infants are shown in the table.

On admission the severity of illness of the infants in the INF- $\alpha-2$ a group was significantly greater than that of the placebo group. However, more rapid drop of the clinical score after the second day of treatment was observed in the INF- $\alpha-2 a$ treated group $(-6.63 v$ $-5.09, p=0.05$ by Wilcoxon rank sum test) and the mean increment of improvement in the illness score between admission and day 3 was also greater in the infants treated with INF- $\alpha-2 a(-8.93 v-7.00, p=0.02$; fig 1$)$. There were no significant differences in the changes of temperature, respiratory rate, and pulse rate between the two groups.

The initial determination of the $\mathrm{SaO}_{2}$ showed that eight infants of the INF- $\alpha-2 a$ treated group and 14 infants of the placebo

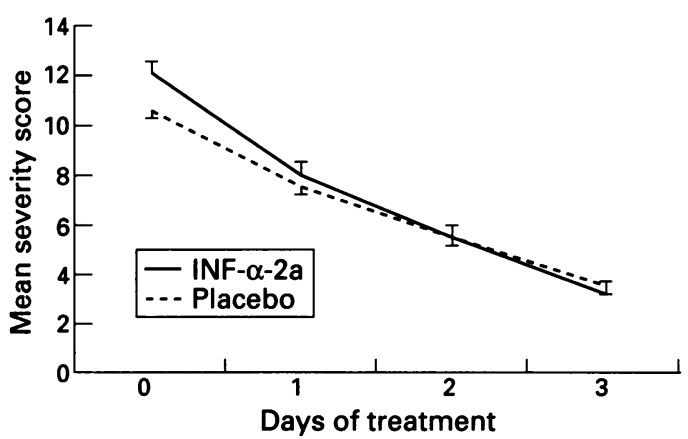

Figure 1 Means of daily scores for severity of illness in patients treated with INF- $\alpha-2 a$ and placebo.

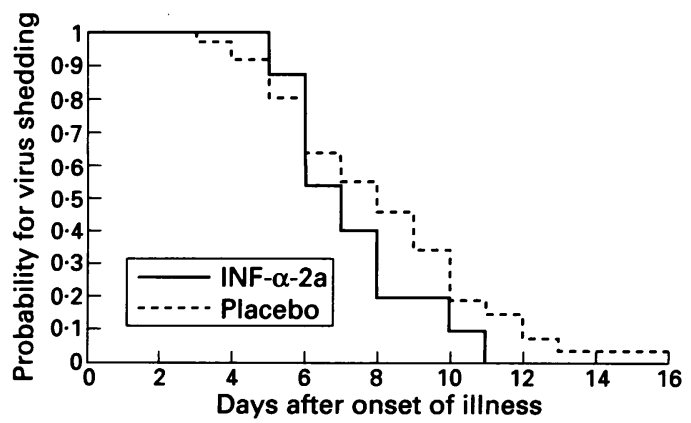

Figure 2 Kaplan-Meier curves for duration of virus shedding time after onset of illness in patients with respiratory syncytial virus bronchiolitis treated with INF- $\alpha-2 a$ and placebo. 
group had an $\mathrm{SaO}_{2}$ less than $92 \%$ and oxygen treatment was given to these infants. The mean (SD) duration of oxygen treatment was similar in the two groups (INF- $\alpha-2 \mathrm{a}: 2.88(1.05)$ days $v$ placebo: $3.36(1.59)$ days). None of the patients had respiratory failure or required assisted ventilation.

The respiratory syncytial virus shedding time estimated by the Kaplan-Meier life table method showed that $50 \%$ of the patients in the INF- $\alpha-2 a$ treated group stopped shedding respiratory syncytial virus seven days after onset of illness compared with eight days in the placebo group (fig 2). This difference, however, was not statistically significant $(p=0.26$ by Mantel-Cox log rank test).

No toxicity or side effects from the INF- $\alpha-2 a$ treatment were noted in any infant. Complete blood cell counts and hepatic transaminase values obtained before and at the end of treatment were not significantly different between the placebo and INF- $\alpha-2 a$ groups, and no haematological abnormality was noted. All 52 infants recovered from their respiratory syncytial virus infection. The duration of hospital stay ranged from four to 12 days with a mean of $6.25(1.73)$ days. The duration of hospital stay was not further compared in the two groups as uniform discharge criteria were not applied during the study period. Two patients from the INF- $\alpha-2 a$ group and four patients from the placebo group respectively stayed in the hospital for more than eight days because of prolonged tachypnoea or wheezing.

\section{Discussion}

It is known that although respiratory syncytial virus is very sensitive to the action of interferon in vitro, ${ }^{10}$ it is a poor inducer of interferon in human cells. ${ }^{11} 12$ This paradox suggested that INF- $\alpha$ might be an effective agent for the treatment of respiratory syncytial virus infection. The findings of our study showed that in the first three days of admission infants treated with INF- $\alpha-2 a$ did have significantly more rapid drop of clinical severity score when compared with infants receiving placebo. However, in both groups patients were equally well after day 3 and no differences of hospital stay were observed, whether the greater relative magnitude of improvement in the first three days of treatment is clinically relevant is not clear. The clinical course and outcome of acute bronchiolitis in Hong Kong infants is in general benign ${ }^{8}$ and most of our study cases had a mild to moderate degree of disease severity, and none of the patients had respiratory failure. It is therefore difficult to predict whether INF- $\alpha$ treatment would have more obvious effects in more severe cases to the degree that would prevent respiratory failure and decrease the length of hospital stay.

No difference of the viral shedding time between the INF- $\alpha-2 a$ and placebo groups was observed in this study. Three different methods of detecting the viral agents were employed to ensure the test was sufficiently sensitive. Whether the negative result in this respect was related to the small case number is unknown. A study with larger case numbers or increased dosage of INF- $\alpha$ would be worthwhile.

A parenteral preparation of $\mathrm{NNF}-\alpha$ was chosen for this study because of its ready availability and simple method of administration. Nevertheless, further trials with nebulised $\mathrm{NNF}-\alpha$ are warranted because of its expected direct effect in the treatment of respiratory tract infection.

1 Sung RYT, Murray HGS, Chan RCK, Davies DP, French GL. Seasonal patterns of respiratory syncytial virus infection in Hong Kong: a preliminary report. $\mathcal{F}$ Infect Dis 1987 156: 527-8.

2 Laufer FS, Edelson PJ. Respiratory syncytial virus infection and cardiopulmonary disease. Pediatr Ann 1987; 16: and -53 .

3 Kim HW, Canchola JG, Brandt DC, et al. Respiratory syncytial virus disease in infants despite prior administration of antigenic inactivated vaccine. Am $\Im$ Epidemiol 1969; 89: of antigenic

4 Committee on infectious diseases American Academy of Pediatrics. Ribavirin therapy of respiratory syncytial virus. Pediatrics 1987; 79: 475-7.

5 Isaacs D. Production of interferon in respiratory syncytial virus bronchiolitis. Arch Dis Child 1989; 64: 92-5.

6 Taylor CE, Webb MSC, Milner AD, et al. Interferon alfa, infectious virus, and virus antigen secretion in respiratory syncytial virus infections of graded severity. Arch Dis Child 1989; 64: 1656-60.

7 Portnoy J, Hicks R, Pachecs F, et al. Pilot study of recombinant interferon alpha-2a for the treatment of infants with nant interferon alpha-2a for the treatment of infants with bronchiolitis induced by respiratory syncytial

8 Sung RYT, Chan RCK, Tam TS, Cheng AFB, Murray HGS. Epidemiology and aetiology of acute bronchiolitis in Hong Kong infants. Epidemiol Infect 1992; 108: 147-54.

9 Lauer BA, Masters HA, Wren CG, Leurin MJ. Rapid detection of respiratory syncytial virus in nasopharyngeal secretions by enzyme-linked immunosorbent assay. $₹ \mathrm{Clin}$ Microbiol 1985; 22: 782-5.

10 Moching JM, Forsyth BR. The role of the interferon system in respiratory syncytial virus infections. Proc Soc Exp Biol Med 1971; 138: 1009-14.

11 Hall CB, Douglas RG Jr, Simons RL, Geiman JM. Interferon production in infants with respiratory syncytial, influenza and parainfluenza virus infections $\mathcal{F}$ Pedia 1978; 93: 28-32.

12 Gardner PS, McGuckin R, Beale AJ, Fernandes R. Interferon and respiratory syncytial virus. Lancet 1970; i: 574-5. 